\title{
Segurança de barragens e os riscos potenciais à saúde pública
}

\section{Safety of dams and the potential risks to public health}

\author{
Eliane Lima e Silva', Mariano Andrade da Silva²
}

DOI: 10.1590/0103-11042020E217

\begin{abstract}
RESUMO Este artigo visou refletir sobre a implementação e a efetividade da Política Nacional de Segurança de Barragens (PNSB) e sobre as ações de redução do risco de desastres no contexto da saúde pública. As barragens podem ter diversos usos, e seu rompimento causa danos sociais, ambientais e sanitários de grande dimensão que perduram ao longo do tempo. O estudo foi realizado a partir de revisão da literatura e análise de dados do cadastro de barragens no Brasil de 2017. Hoje, não se sabe o quantitativo de barragens existentes nem as reais estatísticas das ocorrências de incidentes/acidentes por danos ou colapso das estruturas. São vários os atores envolvidos no processo de segurança de barragens, e 43 órgãos têm a atribuição de fiscalizar mais de 24 mil barragens cadastradas no Sistema Nacional de Informações sobre Segurança de Barragens, em que 76\% dos registros não possuem informações suficientes para definir sua submissão à PNSB. Os impactos sobre a saúde, pessoas e serviços são diversos, diretos e indiretos. Assim, o trabalho procura demostrar que a PNSB é um dispositivo com potencialidade mudar o cenário de gestão de risco de desastre, e sua efetiva implementação promoverá a redução das incertezas quanto ao risco à população.
\end{abstract}

PALAVRAS-CHAVE Desastres. Barragem. Risco à saúde humana. Saúde ambiental.

ABSTRACT This article aimed to reflect on the implementation and effectiveness of the National Dam Safety Policy (PNSB) and on disaster risk reduction actions in the context of public health. Dams can have several uses, and their rupture causes great social, environmental, and health damage that lingers over time. The study was carried out based on a literature review and data analysis of the dam registry in Brazil in 2017. Today, the number of existing dams and the real statistics on the occurrence of incidents/accidents due to damage or collapse of structures are not known. There are several actors involved in the dam safety process, and 43 bodies are responsible for inspecting more than 24,000 dams registered in the National Dam Safety Information System, in which $76 \%$ of the records do not have enough information to define their submission to the PNSB. The impacts on health, people, and services are diverse, direct, and indirect. Thus, this work seeks to demonstrate that the PNSB is a device with the potential to change the scenario of disaster risk management, and its effective implementation will promote the reduction of uncertainties regarding risk to the population.

KEYWORDS Disasters. Dam. Health risk. Environmental health.
1 Universidade de Brasília (UnB), Departamento de Geografia (GEA), Laboratório de Geografia, Ambiente e Saúde (Lagas) - Brasília (DF), Brasil. elianelima26@gmail.com

2 Fundação Oswaldo Cruz (Fiocruz), Escola Naciona de Saúde Pública Sergio Arouca (Ensp), Centro de Estudos e Pesquisas em Emergências e Desastres em Saúde (Cepedes) - Rio de Janeiro (RJ), Brasil. 


\section{Introdução}

Barragem é definida como qualquer estrutura em um curso permanente ou temporário de água para fins de contenção ou acumulação de substâncias líquidas ou misturas de líquidos e sólidos, compreendendo o barramento e as estruturas associadas $\mathbf{1}$. Sua importância se ampliou ao longo da história contribuindo para o controle de inundações, produção de eletricidade, disponibilização de água para consumo humano, para fins comerciais ou recreação, e ainda na atividade de mineração para a contenção de rejeitos ${ }^{\mathbf{1}}$.

No Brasil, a elaboração dos projetos, a construção e a manutenção das barragens seguem normativas e critérios distintos, o que reduz seu fator de confiança'. O colapso dessas barragens pode causar danos ao ambiente e à propriedade, provocando diversos impactos, diretos e indiretos, que podem perdurar por longo tempo. Em sua vida útil, a infraestrutura das barragens pode se desgastar ampliando o risco de rompimento e, com isso, o risco à vida.

Exemplos recentes no Brasil foram observados com o Desastre da Samarco, em 2015, e o da Vale, em 2019, que atingiram diretamente os municípios de Mariana e Brumadinho, Minas Gerais, além de muitos outros municípios, ultrapassando até o limite do estado ${ }^{2}$. Essas são as ocorrências que ganharam visibilidade pelo seu impacto imediato, mas não foram as únicas registradas no Brasil.

Considerando o risco oferecido pelas barragens que estão distribuídas no País, em 2010, foi instituída a Política Nacional de Segurança de Barragem (PNSB), que estabeleceu um conjunto de atribuições e responsabilidades além de instrumentos para a gestão do risco de rompimento dessas estruturas ${ }^{3}$. Passados nove anos da entrada em vigor da PNSB, a sua implementação ainda não é efetiva, além de envolver muitas instituições distintas, com competências federais, estaduais e municipais.

Nesse contexto, o objetivo deste artigo é refletir sobre a implementação e a efetividade da PNSB e sobre as ações de Redução do Risco de Desastres (RRD) no contexto da saúde pública.

\section{Material e métodos}

Os resultados e reflexões deste artigo foram alcançados a partir de dados secundários para análise do cenário de risco de rompimento de barragens no Brasil.

Para isso, em princípio, foi realizado um levantamento de publicações relevantes para o tema, tanto de artigos científicos quanto de normativas e documentos técnicos produzidos por instituições governamentais diretamente envolvidas na implementação da PNSB.

A distribuição espacial e a caracterização das barragens foram realizadas a partir de dados do Sistema Nacional de Informações sobre Segurança de Barragens (Snisb), do ano de 2017, inicialmente observando o cadastro geral de todas as barragens e, em seguida, analisando apenas as estruturas com Classificação de Risco (CRI) e Dano Potencial Associado (DPA) altos, em virtude de grandes impactos a eles atribuíveis em situação de rompimento, econômicos, sociais, ambientais ou de perda de vidas humanas, incluindo aqueles que incidem direta e indiretamente sobre a saúde pública.

Para análise dos dados e informações, estes foram tabulados, geocodificados e distribuídos espacialmente utilizando os softwares Excel $^{\circledR}$ e Quantum Gis ${ }^{\circledast}$ (QGIS).

\section{Resultados e discussão}

O estabelecimento de medidas que obriguem empresas que exploram atividades que envolvem barragens, em seus usos diversos, e o seu efetivo cumprimento têm se apresentado como um desafio, não apenas no Brasil, mas em diversos outros países. Nações como os EUA, o Canadá e a Austrália foram precursores em normatizar a segurança de barragem. O Brasil estabeleceu uma política para isso apenas em $2010^{\mathbf{1 , 4}}$.

Rompimentos de barragens alteram aspectos ecológicos, econômicos e sociais e reduzem ou até eliminam a confiança das pessoas nas empresas e nas instituições governamentais. Entre os fatores que contribuem ou que são 
a causa de comprometimento ou colapso das estruturas das barragens, estão: o projeto ou a localização inadequada, processo de construção e de operação negligenciados levando ao comprometimento da estabilidade física ao longo do tempo ${ }^{4-6}$.

Não se pode afirmar o quantitativo de barragens existentes e as reais estatísticas das ocorrências de incidentes e acidentes em decorrência de danos ou colapso na estrutura das barragens. Tanto em âmbito internacional quanto nacional, são vários os atores envolvidos no processo de segurança de barragens, incluindo instituições governamentais e não governamentais.

O processo de reconstrução após a Segunda Guerra Mundial gerou grande demanda por matéria prima e culminou na ampliação da atividade de mineração.

$\mathrm{O}$ aumento dos preços das commodities nos anos 2000 levou à reabertura de minas, Wanderley et al. ${ }^{5}$. apontaram que, entre 2003 e 2013 , houve um aumento de $630 \%$ nas importações globais de minérios, no entanto, as exportações tinham origem em poucos países sendo, em 2013, o Brasil o responsável por $14,3 \%$ das exportações mundiais dessa commodities, o segundo maior exportador do mundo ${ }^{5}$.

Com a ampliação dessa demanda, no decorrer do tempo, observou-se que ocorrências de acidentes diminuíram em barragens de pequeno a médio porte e aumentaram nas de grande porte, de $29 \%$ para $40 \%$, quando comparadas a ocorrências anteriores e posteriores ao ano $2000^{7}$.

\section{Política Nacional de Segurança de Barragens (PNSB)}

A PNSB, instituída pela Lei n ${ }^{\circ} 12.334 / 2010$,é aplicada a barragens destinadas à acumulação de água para quaisquer usos, à disposição final ou temporária de rejeitos e à acumulação de resíduos industriais ${ }^{3}$.

Aplica-se a estruturas que possuam altura do maciço maior ou igual a 15 metros, contados do ponto mais baixo da fundação à crista, capacidade total de armazenamento maior ou igual a 3 milhões de metros cúbicos, reservatório com resíduos perigosos e categoria de dano potencial (econômicos, sociais, ambientais ou de perda de vidas humanas) associado médio ou alto ${ }^{3}$.

A lei estabelece que a PNSB deve ser considerada desde sua concepção até seu descomissionamento e usos futuros (planejamento, projeto, construção, primeiro enchimento e primeiro vertimento, operação, desativação) e que ações preventivas e emergências devem prever envolvimento direto ou indireto da população. Destaca ainda que o empreendedor é o responsável pela segurança, que mecanismos de controle social devem ser promovidos e que a segurança de uma barragem é parte de sua sustentabilidade e dos potenciais efeitos sociais e ambientais ${ }^{3}$.

Apresentam-se sete instrumentos da política: a) CRI e DPA; b) Plano de Segurança; c) Snisb; d) Sistema Nacional de Informações sobre o Meio Ambiente (Sisnama); e) Cadastro Técnico Federal de Atividades e Instrumentos de Defesa Ambiental; f) Cadastro Técnico Federal de Atividades Potencialmente Poluidoras ou Utilizadoras de Recursos Ambientais; g) Relatório de Segurança de Barragens (RSB).

Para a implementação da PNSB, diversos são os atores envolvidos, com destaque para os órgãos fiscalizadores e os empreendedores.

\section{Estrutura e funcionamento da PNSB}

Vale destacar que a PNSB, quando institui o Snisb, estabelece que a fiscalização da segurança de barragens caberá, sem prejuízo das ações fiscalizatórias dos órgãos ambientais integrantes do Sisnama:

- à entidade que outorgou o direito de uso dos recursos hídricos, observado o domínio do corpo hídrico, quando o objeto for de acumulação de água, exceto para fins de aproveitamento hidrelétrico;

- à entidade que concedeu ou autorizou o 
uso do potencial hidráulico, quando se tratar de uso preponderante para fins de geração hidrelétrica;

- à entidade outorgante de direitos minerários para fins de disposição final ou temporária de rejeitos;

- à entidade que forneceu a licença ambiental de instalação e operação para fins de disposição de resíduos industriais.

Assim, esses atores têm a responsabilidade, guardadas as devidas competências, em todo território nacional, de realizar a coleta, o tratamento, o armazenamento e a recuperação de informações sobre barragem, devendo contemplar desde barragens em construção às que estão em operação ou desativadas, uma vez que, por princípio de funcionamento, o cadastramento das barragens no Snisb é descentralizado e a coordenação geral do sistema cabe à Agência Nacional das Águas (ANA).

A ANA é responsável por articular os órgãos fiscalizadores na implementação da PNSB; organizar, implantar e gerir o Snisb, que envolve desde a concepção das estratégias e diretrizes até sua disponibilização para os atores diretamente envolvidos na temática e para o público em geral; coordenar a elaboração do RSB; e fiscalizar e monitorar em conjunto com agências estaduais de recursos hídricos a segurança das barragens de usos múltiplos. A Agência Nacional de Energia Elétrica (Aneel) é responsável pela fiscalização das barragens hidrelétricas. O Ibama e órgãos ambientais estaduais são responsáveis pela fiscalização das barragens de resíduos industriais. A Agência Nacional de Mineração (ANM), responsável pela fiscalização das barragens de mineração, acumula tanto a função outorgante de direitos minerários para fins de disposição final ou temporária desses rejeitos quanto a fiscalização e o monitoramento dessas barragens. Os proprietários de barragens devem executar ações de segurança e elaborar plano de segurança.

Hoje podem ser identificados 31 órgãos efetivamente fiscalizadores de barragens, que, quando somados aos órgãos potencialmente fiscalizadores, resultam em 43 instituições com essa atribuição, conforme quadro 1.

Quadro 1. Órgãos fiscalizadores e potencialmente fiscalizadores de barragens no Brasil

\begin{tabular}{|c|c|c|}
\hline UF & Órgão Fiscalizador & Potencial Fiscalizador \\
\hline \multicolumn{3}{|c|}{ Âmbito Federal } \\
\hline União & $\begin{array}{l}\text { Agência Nacional das Águas: Fiscaliza a segurança } \\
\text { das barragens de usos múltiplos localizadas em rios } \\
\text { federais } \\
\text { Agência Nacional de Energia Elétrica: Fiscaliza a } \\
\text { segurança das barragens de usinas hidrelétricas } \\
\text { Agência Nacional de Mineração: Fiscaliza a seguran- } \\
\text { ça das barragens de rejeitos de mineração }\end{array}$ & $\begin{array}{l}\text { Instituto Brasileiro do Meio Ambiente e dos Recur- } \\
\text { sos Naturais Renováveis: Fiscaliza a segurança das } \\
\text { barragens de resíduos industriais em rios federais }\end{array}$ \\
\hline \multicolumn{3}{|c|}{ Âmbito Estadual } \\
\hline$A C$ & Instituto de Meio Ambiente do Acre - Imac/AC & \\
\hline$A L$ & $\begin{array}{l}\text { Secretaria de Estado do Meio Ambiente e dos Recur- } \\
\text { sos Hídricos - SEMARH/AL }\end{array}$ & $\begin{array}{l}\text { Instituto do Meio Ambiente do Estado do Alagoas } \\
\text { - IMA/AL }\end{array}$ \\
\hline AM & $\begin{array}{l}\text { Instituto de Proteção Ambiental do Estado do Ama- } \\
\text { zonas - Ipaam/AM }\end{array}$ & \\
\hline AP & $\begin{array}{l}\text { Instituto do Meio Ambiente e de Ordenamento Terri- } \\
\text { torial do Estado do Amapá - Imap/AP }\end{array}$ & \\
\hline BA & $\begin{array}{l}\text { Instituto do Meio Ambiente e Recursos Hídricos - } \\
\text { Inema/BA }\end{array}$ & \\
\hline
\end{tabular}


Quadro 1. (cont.)

\begin{tabular}{|c|c|c|}
\hline UF & Órgão Fiscalizador & Potencial Fiscalizador \\
\hline CE & Secretaria dos Recursos Hídricos - SRH/CE & $\begin{array}{l}\text { Secretaria do Meio Ambiente do Estado do Ceará - } \\
\text { Semace/CE }\end{array}$ \\
\hline DF & $\begin{array}{l}\text { Agência Reguladora de Águas, Energia e Saneamen- } \\
\text { to Básico do Distrito Federal - Adasa/DF }\end{array}$ & Instituto Brasília Ambiental - Ibram/DF \\
\hline ES & Agência Estadual de Recursos Hídricos - AGERH/ES & $\begin{array}{l}\text { Instituto Estadual de Meio Ambiente e Recursos } \\
\text { Hídricos - lema/ES }\end{array}$ \\
\hline GO & $\begin{array}{l}\text { Secretaria de Meio Ambiente, Recursos Hídricos, } \\
\text { Infraestrutura, Cidades e Assuntos Metropolitanos } \\
\text { - Secima/GO }\end{array}$ & \\
\hline MA & $\begin{array}{l}\text { Secretaria de Estado de Meio Ambiente e Recursos } \\
\text { Naturais do Maranhão - Sema/MA }\end{array}$ & \\
\hline MG & $\begin{array}{l}\text { Secretaria de Estado de Meio Ambiente e Desenvol- } \\
\text { vimento Sustentável - Semad/MG }\end{array}$ & \\
\hline MT & Secretaria de Estado do Meio Ambiente - Sema/MT & \\
\hline MS & $\begin{array}{l}\text { Instituto de Meio Ambiente de Mato Grosso do Sul } \\
\text { - Imasul/MS }\end{array}$ & \\
\hline PA & $\begin{array}{l}\text { Secretaria de Meio Ambiente e Sustentabilidade - } \\
\text { Semas/PA }\end{array}$ & \\
\hline PB & $\begin{array}{l}\text { Agência Executiva de Gestão das Águas do Estado } \\
\text { da Paraíba - Aesa/PB }\end{array}$ & $\begin{array}{l}\text { Superintendência de Administração do Meio Am- } \\
\text { biente - Sudema/PB }\end{array}$ \\
\hline$P E$ & $\begin{array}{l}\text { Agência Pernambucana de Águas e Clima - Apac/ } \\
\text { PE }\end{array}$ & Agência Estadual do Meio Ambiente - CPRH/PE \\
\hline PI & $\begin{array}{l}\text { Secretaria estadual do Meio Ambiente e Recursos } \\
\text { Hídricos - Semar/PI }\end{array}$ & \\
\hline PR & Instituto das Águas do Paraná - Águas Paraná/PR & Instituto Ambiental do Paraná - IAP/PR \\
\hline RJ & Instituto Estadual do Ambiente - Inea/RJ & \\
\hline $\mathrm{RN}$ & $\begin{array}{l}\text { Instituto de Gestão das Águas do Estado do Rio } \\
\text { Grande do Norte - IGARN/RN }\end{array}$ & $\begin{array}{l}\text { Instituto de Desenvolvimento Sustentável e Meio } \\
\text { Ambiente - Idema/RN }\end{array}$ \\
\hline $\mathrm{RO}$ & $\begin{array}{l}\text { Secretaria de Estado do Desenvolvimento Ambiental } \\
\text { - Sedam/RO }\end{array}$ & \\
\hline RR & $\begin{array}{l}\text { Fundação Estadual do Meio Ambiente e Recursos } \\
\text { Hídricos - FEMARH/RR }\end{array}$ & \\
\hline RS & $\begin{array}{l}\text { Departamento de Recursos Hídricos da Secretaria do } \\
\text { Ambiente e Desenvolvimento Sustentável - DRH- } \\
\text {-Sema/RS }\end{array}$ & $\begin{array}{l}\text { Fundação Estadual de Proteção Ambiental - Fepam/ } \\
\text { RS }\end{array}$ \\
\hline SC & $\begin{array}{l}\text { Secretaria de Estado do Desenvolvimento Econômi- } \\
\text { co Sustentável - SDS/SC }\end{array}$ & Instituto do Meio Ambiente - IMA/SC \\
\hline SE & $\begin{array}{l}\text { Secretaria de Estado do Meio Ambiente e dos Recur- } \\
\text { sos Hídricos - SEMARH/SE }\end{array}$ & $\begin{array}{l}\text { Administração Estadual do Meio Ambiente - Ade- } \\
\mathrm{ma} / \mathrm{SE}\end{array}$ \\
\hline SP & $\begin{array}{l}\text { Departamento de Águas e Energia Elétrica - DAEE/ } \\
\text { SP } \\
\text { Companhia Ambiental do Estado de São Paulo - Ce- } \\
\text { tesb/SP }\end{array}$ & \\
\hline TO & Instituto Natureza de Tocantins - Naturatins/TO & \\
\hline
\end{tabular}

Fonte: Dados - ANA, $2018^{8}$ 
O último RSB, divulgado pela ANA, em 2018, apresenta dados que levam à reflexão sobre a implementação da PNSB. O plano de segurança e sua revisão periódica foram regulamentados por $65 \%$ dos órgãos fiscalizadores, enquanto $62,5 \%$ fizeram inspeções regulares, e $51 \%$ realizaram inspeções especiais. Apenas $45 \%$ apresentaram plano de emergência, conforme estabelece os arts. 11 e 12 da lei. Outro aspecto relevante apontado pela ANA é que, até 2017, oito desses órgãos não tinham regulamentado a PNSB, fator imprescindível para que empreendedores saibam o que deve ser feito em suas barragens para atendimento às normativas ${ }^{8}$.

A classificação das barragens se dá conforme suas características estruturais (altura) e volume do seu reservatório bem como os danos potenciais caso haja a sua ruptura. Barragens de grande porte são aquelas que possuem mais de 15 metros de altura ou qualquer barragem com altura de 5 metros a 15 metros em que o comprimento da crista seja maior que 500 metros e/ou que o volume do reservatório maior que 3 milhões de metros cúbicos e/ou a capacidade de descarga maior que $2 \mathrm{mil} \mathrm{m}^{3} / \mathrm{s}$ e/ou barragens com fundações pouco usuais ${ }^{3}$.

\section{Cenário de barragens no Brasil: um olhar sobre a classificação e distribuição espacial}

No Snisb, existem 24.092 barragens de usos diversos cadastradas no Brasil ( figura 1); e, apesar de $97 \%$ delas possuir identificação do empreendedor, em 76\% não há informações suficientes para definir sua submissão ou não à $\mathrm{PNSB}^{8}$.

Figura 1. Distribuição espacial das barragens no Brasil, 2017

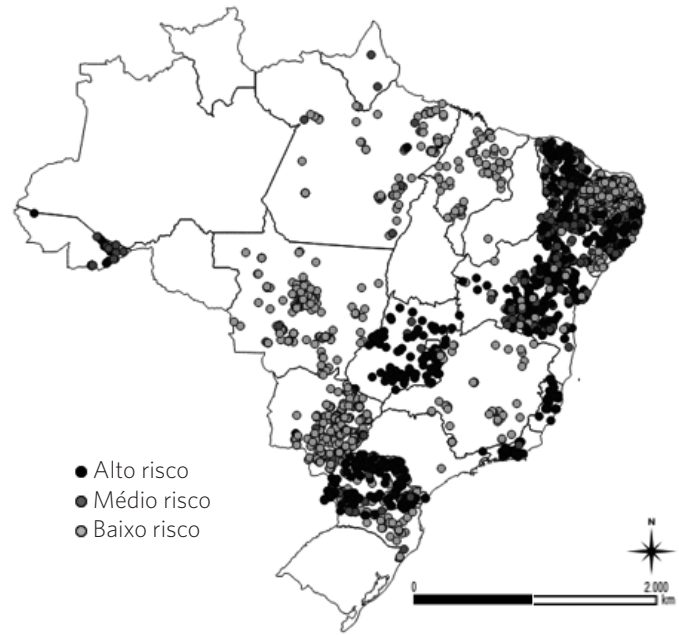

Fonte: Elaboração própria a partir de dados do Snisb/ANA, 20178

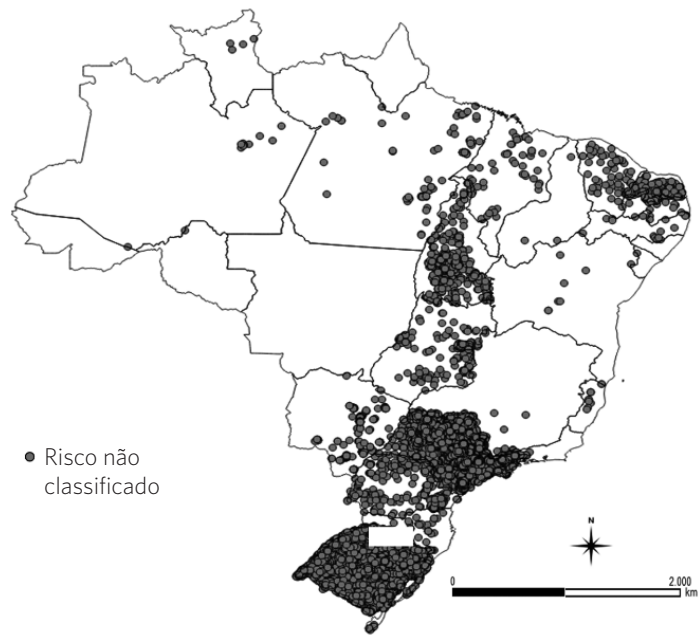

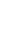


econômicos e ambientais que podem causar. Esses indicadores foram estabelecidos para orientar reguladores e fiscalizadores da segurança de barragens. A situação dessas barragens no Snisb (figura 1) é que não se sabe o risco em $85,3 \%$ delas por ter apenas o cadastro e não possuir informações que possibilite estabelecer o grau de risco, enquanto 7,3\% são de baixo, $2,8 \%$ de médio e $4,7 \%$ de alto risco' ${ }^{1}$.

Nesse cenário, não é possível estabelecer a gestão do risco de barragens uma vez que não se sabe a real situação de ameaça que elas representam. A classificação da barragem é essencial para efetivar a implementação da PNSB, e o estabelecimento da CRI e do DPA é parte dos critérios para definir se uma estrutura se submete ou não à PNSB.

Os princípios estabelecidos nos instrumentos internacionais pactuados para a RRD apontam que o processo de desenvolvimento (econômico, social e tecnológico) deve observar os aspectos de sustentabilidade ambiental e contribuir para a redução das vulnerabilidades sociais, pautados por um olhar multidisciplinar e intersetorial em um contexto multirriscos ${ }^{22}$. Incorporar esse olhar no processo de trabalho dos entes envolvidos na implementação da PNSB apresenta-se como um desafio.

\section{Barragens com alto risco de rompimento e alto dano potencial associado no Brasil}

A incerteza quanto ao risco real permanece quando analisadas as barragens classificadas quanto à CRI e ao DPA (tabela 1). O primeiro ponto que preocupa é a ausência de informação uma vez que 20.554 (85\%) barragens não são classificadas quanto ao risco, e 18.638 (74\%), quanto ao dano; e daquelas que possuem alto CRI (1124), 228 não têm estabelecido qual o DPA. Quando combinadas alto risco e alto dano, identificam-se 723 estruturas nessa situação.

Tabela 1. Número de barragens segundo categoria de risco e dano potencial associado no Brasil, 2017

\begin{tabular}{lrrrrrr}
\hline Dano potencial associado & \multicolumn{1}{c}{ Maixo } & $\begin{array}{r}\text { Não } \\
\text { Classificada }\end{array}$ & Total & $\%$ \\
\hline Categoria de risco & Alto & Médio & Bar & & & \\
\hline Alto & 723 & 71 & 102 & 228 & 1.124 & 4,7 \\
Médio & 298 & 129 & 242 & 1 & 670 & 2,8 \\
Baixo & 812 & 229 & 707 & 1 & 1.749 & 7,3 \\
Não Classificada & 1.153 & 260 & 733 & 18.408 & 20.554 & 85,3 \\
Total & 2.986 & 689 & 1.784 & 18.638 & 24.097 & 100,0 \\
$\%$ & 12,4 & 2,9 & 7,4 & 77,3 & 100,0 & - \\
\hline
\end{tabular}

Fonte: Elaboração própria a partir de dados do Snisb/ANA10.

Essas estruturas estão mais concentradas no nordeste brasileiro, em que Rio Grande do Norte (219), Bahia (187) e Paraíba (167) concentram maior número, prevalecendo o uso para abastecimento de água e irrigação (figura 2). 
Figura 2. Distribuição espacial das barragens com alto risco de rompimento e alto dano potencial, no Brasil, 2017

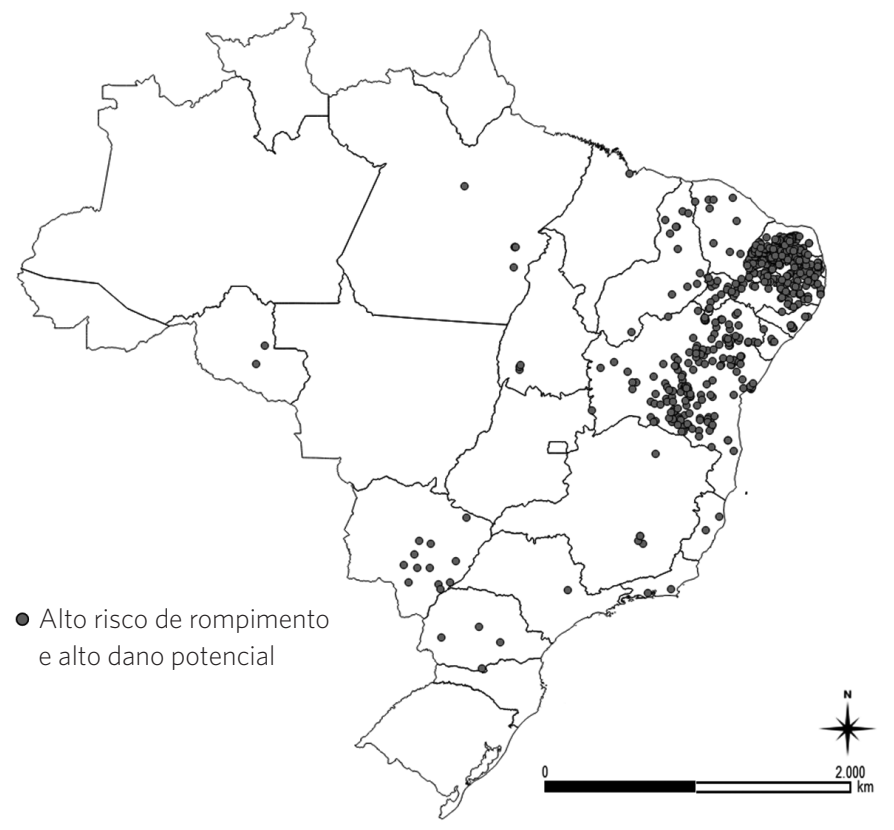

Fonte: Elaboração própria a partir de dados do Snisb/ANA10.

De todas as barragens, $42 \%$ (24 mil) não possuem nenhum ato de autorização, outorga ou licenciamento; e, em $76 \%$ dos casos, foi definido se a barragem é ou não submetida à PNSB, também por ausência de informação. Cabe questionar se é possível conhecer o contexto de risco e em que medida as pessoas estão potencialmente expostas uma vez que há um cenário de incerteza no País.

Estudos demonstram que falhas em barragens ocorrem principalmente naquelas com alturas de até 30 metros, e isso corrobora o estabelecido na PNSB quando utiliza a dimensão como variável considerada na estimativa de risco e danos. Azam e $\mathrm{Li}^{7}$ compararam acidentes pré-2000 e pós-2000 e identificaram que falhas em barragens de até 15 metros de altura aumentaram de $28 \%$ para $30 \%$, enquanto falhas entre 15 metros e 30 metros aumentaram de $21 \%$ para $60 \%$ entre os dois períodos. Eles atribuem esse crescimento a uma combinação de construção rápida de diques com monitoramento deficiente ${ }^{7}$.

\section{Acidentes e desastres por rompimento de barragens}

O desenvolvimento de técnicas para construção de barragens de contenção de rejeitos ocorreu de forma empírica, utilizando-se da experiência dos mineiros e sem o uso de técnicas de engenharia de barragens, principalmente em países em desenvolvimento.

Azam e $\mathrm{Li}^{7}$ analisaram 218 falhas de barragens de rejeitos no mundo, entre 1910 e 2009, apresentando uma análise espaço-temporal que identificou que as décadas de 1960, 1970 e 1980 registraram cerca de 50 eventos cada - América do Norte (36\%), Europa (26\%) e América do Sul (19\%). Entre 2000 e 2009, foram registrados 20 acidentes na Europa e Ásia, e atribui-se essa redução à evolução da tecnologia e à adoção de critérios de segurança ${ }^{7}$.

Os principais fatores que estão associados às causas de rompimento de barragens são problemas de fundação, capacidade inadequada dos vertedouros, instabilidade dos taludes, 
erosões, deficiência no controle e inspeção pós-operação e ausência ou inadequação de procedimentos de segurança ao longo da vida útil da estrutura. Ainda nesse contexto, Azam e Li analisaram as falhas nas barragens, identificando que aquelas associadas a chuvas intensas passaram de $25 \%$ antes de 2000 para $40 \%$ após 2000. Comportamento similar ocorreu em decorrência de má gestão, passando de $10 \%$ para $30 \%$. Outro aspecto relevante apontado é que, geograficamente, os acidentes passaram de países desenvolvidos para países em desenvolvimento ${ }^{5,7}$.

Davies e Martin 9 corroboram a percepção de aumento dos rompimentos de barragens de mineração e apontam que a demanda mundial por minérios levou à rápida ampliação da capacidade de produção, atribuindo que alguns aspectos nessa iniciativa influenciaram os colapsos, em que destacam: a pressa para iniciar a operação em detrimento da qualidade da execução dos projetos; a pressão sobre órgãos ambientais para acelerar o processo de licenciamento; a contratação de profissionais menos experientes em virtude do aumento dos custos dos serviços e a escassez de mão de obra qualificada no mercado, além da intensificação da produção e pressão por redução nos custos a partir do momento de retração dos preços ${ }^{9}$.

Rico et al. ${ }^{10}$, ao analisarem 147 incidentes de grande porte envolvendo barragens de mineração, apontam que o fator organizacional combinado aos fatores meteorológico são as principais causas relacionadas com as falhas registradas. Do número total, $83 \%$ ocorreram quando a barragem estava ativa; $15 \%$, em barragens inativas e abandonadas; e apenas $2 \%$ das falhas ocorreram em barragens inativas, porém com manutenção ${ }^{\mathbf{1 0}}$.

Bowker e Chambers, que analisaram acidentes com barragens de rejeitos registrados entre 1910 e 2010, apontaram alteração no comportamento dos eventos quanto ao impacto, identificando, em todo o mundo, mais de 30 rompimentos após a década de 1990 que apresentaram consequências graves ou muito graves. Levantam a hipótese que isso pode ser reflexo do uso de tecnologias modernas de mineração, que possibilitam a implantação de megaminas, construídas para extrair minérios a partir de reservas caracterizadas por concentrações minerais cada vez menores. A diminuição da qualidade dos minérios extraídos aumenta a quantidade de rejeitos e o tamanho das barragens $s^{5,11}$.

Com o avanço da tecnologia e a ampliação do volume de extração de minérios, as barragens, que antes eram rudimentares e de pequeno porte, passaram a armazenar uma quantidade maior de rejeitos, registrando-se colapsos com impactos significativos ${ }^{\mathbf{4}, 6}$. Uma característica deste tipo de estruturas está relacionada com a elevada frequência com que as barragens de rejeito falharam, apresentando taxa significativamente mais alta do que a taxa de falhas nas barragens de reservatórios de abastecimento de água ${ }^{\mathbf{1 2}}$.

Quando se discutem rompimentos de barragens no Brasil, a ênfase incide sobre o Desastre da Samarco e da Vale, em Mariana e Brumadinho, ambos em Minas Gerais. No entanto, entre os anos de 1986 e 2019, o Brasil registrou 19 acidentes graves envolvendo barragens; decorrentes desses eventos, ao menos 342 pessoas foram a óbito (quadro 2). A partir da publicação da PNSB, quando contabilizados inclusive acidentes e incidentes de menor porte, anualmente, há, em média, três eventos envolvendo barragem, totalizando 24 registros dessa natureza, para o período 2011-20178. 
Quadro 2. Acidentes com barragens no Brasil

\begin{tabular}{|c|c|c|c|c|}
\hline Ano & Empresa & $\begin{array}{l}\text { Tipo de } \\
\text { barragem }\end{array}$ & Município & Breve descrição \\
\hline 1986 & $\begin{array}{l}\text { Mineração Hercu- } \\
\text { lano }\end{array}$ & $\begin{array}{l}\text { Barragem de } \\
\text { Rejeito }\end{array}$ & Itabirito-MG & Rompimento de Barragem: 7 óbitos. \\
\hline 2001 & $\begin{array}{l}\text { Mineração Rio } \\
\text { Verde }\end{array}$ & $\begin{array}{l}\text { Barragem de } \\
\text { Rejeito }\end{array}$ & $\begin{array}{l}\text { Nova Lima- } \\
-M G\end{array}$ & $\begin{array}{l}\text { Rompimento de Barragem: assoreamento do córrego } \\
\text { taquara e } 5 \text { óbitos. }\end{array}$ \\
\hline 2003 & $\begin{array}{l}\text { Indústria Catagua- } \\
\text { ses de Papel }\end{array}$ & $\begin{array}{l}\text { Resíduo indus- } \\
\text { trial }\end{array}$ & $\begin{array}{l}\text { Cataguases- } \\
-M G\end{array}$ & $\begin{array}{l}\text { Rompimento de Barragem de rejeitos industriais } \\
\text { ( } 900 \text { mil } \mathrm{m}^{3} \text { de licor negro): Danos ambientais e } \\
\text { econômicos. }\end{array}$ \\
\hline 2003 & $\begin{array}{l}\text { Barragem de Ca- } \\
\text { mará }\end{array}$ & $\begin{array}{l}\text { Barragem de } \\
\text { uso múltiplos }\end{array}$ & $\begin{array}{l}\text { Alagoa Nova- } \\
\text {-PB }\end{array}$ & $\begin{array}{l}\text { Rompimento de Barragem: } 5 \text { óbitos; } 3 \text { mil desabriga- } \\
\text { das nas cidades de Alagoa Nova, Areia, Mulungu e } \\
\text { Alagoa Grande. }\end{array}$ \\
\hline 2006 & $\begin{array}{l}\text { Rio Pomba Cata- } \\
\text { guases }\end{array}$ & $\begin{array}{l}\text { Resíduo indus- } \\
\text { trial }\end{array}$ & Miraí-MG & $\begin{array}{l}\text { Vazamento de 1,2 milhões de m³: mortandade de } \\
\text { fauna e flora e interrupção de captação de água. }\end{array}$ \\
\hline 2007 & $\begin{array}{l}\text { Rio Pomba Cata- } \\
\text { guases }\end{array}$ & $\begin{array}{l}\text { Resíduo indus- } \\
\text { trial }\end{array}$ & Miraí-MG & $\begin{array}{l}\text { Rompimento de Barragem }\left(2.280 .000 \mathrm{~m}^{3}\right): 4.000 \\
\text { desalojados. }\end{array}$ \\
\hline 2008 & $\begin{array}{l}\text { Cebel (Centrais } \\
\text { elétricas de Belém) } \\
\text { - Usina de Aperta- } \\
\text { dinho }\end{array}$ & $\begin{array}{l}\text { Barragens } \\
\text { hidrelétrica }\end{array}$ & Vilhena-RO & Rompimento de Barragem: danos ambientais. \\
\hline 2008 & CSN & $\begin{array}{l}\text { Barragem de } \\
\text { Rejeito }\end{array}$ & $\begin{array}{l}\text { Congonhas- } \\
-M G\end{array}$ & Rompimento de vertedouro: 40 famílias desalojadas. \\
\hline 2008 & $N / D$ & $\begin{array}{l}\text { Barragem de } \\
\text { Rejeito }\end{array}$ & Itabira-MG & Rompimento de Barragem: danos ambientais. \\
\hline 2009 & $\begin{array}{l}\text { Barragem de Algo- } \\
\text { dões }\end{array}$ & $\begin{array}{l}\text { Barragem de } \\
\text { uso múltiplos }\end{array}$ & $\begin{array}{l}\text { Cocal e Buriti } \\
\text { dos Lopes-PI }\end{array}$ & $\begin{array}{l}\text { Rompimento da Barragem ( } 52 \text { milhões de m³): } 9 \text { óbi- } \\
\text { tos, } 2 \text { mil pessoas desabrigadas e } 953 \text { desalojados, } \\
80 \text { feridos; } 22 \text { comunidades da zona rural de Cocal } \\
\text { afetadas; danos ambientais, econômicos e sociais. }\end{array}$ \\
\hline 2014 & N/D & $\begin{array}{l}\text { Barragem de } \\
\text { uso múltiplos }\end{array}$ & Uruana-GO & Rompimento de Barragem: 2 óbitos. \\
\hline 2014 & $\begin{array}{l}\text { Barreira hidroelétrica } \\
\text { de Santo Antônio }\end{array}$ & $\begin{array}{l}\text { Barragens } \\
\text { hidrelétrica }\end{array}$ & $\begin{array}{l}\text { Laranjal do } \\
\text { Jari-AP }\end{array}$ & Rompimento de Barragem: 4 óbitos. \\
\hline 2014 & $\begin{array}{l}\text { Herculano Mine- } \\
\text { ração }\end{array}$ & $\begin{array}{l}\text { Barragem de } \\
\text { Rejeito }\end{array}$ & Itabirito-MG & Rompimento de Barragem: 3 óbitos. \\
\hline 2015 & Samarco S.A. & $\begin{array}{l}\text { Barragem de } \\
\text { Rejeito }\end{array}$ & Mariana-MG & $\begin{array}{l}\text { Rompimento de Barragem ( } 34 \text { milhões m³): } 19 \text { óbi- } \\
\text { tos ( } 12 \text { terceirizados, } 1 \text { prestador de serviço, } 1 \text { funcio- } \\
\text { nário e } 5 \text { moradores). }\end{array}$ \\
\hline 2016 & $\begin{array}{l}\text { Barragem Fazenda } \\
\text { Guavirova }\end{array}$ & $\begin{array}{l}\text { Barragem de } \\
\text { uso múltiplos }\end{array}$ & $\begin{array}{l}\text { União da } \\
\text { Vitória-PR }\end{array}$ & Rompimento de Barragem: 1 óbitos. \\
\hline 2018 & Barragem rio Uraim & $\begin{array}{l}\text { Barragem de } \\
\text { uso múltiplos }\end{array}$ & $\begin{array}{l}\text { Paragominas- } \\
\text {-PA }\end{array}$ & Rompimento de Barragem: 2 óbitos; 100 desalojados. \\
\hline 2019 & Vale S.A. & $\begin{array}{l}\text { Barragem de } \\
\text { Rejeito }\end{array}$ & $\begin{array}{l}\text { Brumadinho- } \\
-M G\end{array}$ & $\begin{array}{l}\text { Rompimento de Barragem ( } 12 \text { milhões m³): } 248 \\
\text { óbitos e } 22 \text { pessoas desaparecidas. }\end{array}$ \\
\hline 2019 & Barragem do Quati & $\begin{array}{l}\text { Barragem de } \\
\text { uso múltiplos }\end{array}$ & $\begin{array}{l}\text { Pedro Alexan- } \\
\text { dre-BA }\end{array}$ & $\begin{array}{l}\text { Rompimento de Barragem: } 430 \text { famílias ( } 1720 \text { pes- } \\
\text { soas) desalojada/desabrigada. }\end{array}$ \\
\hline
\end{tabular}

Fonte: Elaboração própria a partir de dados do Snisb/ANA 10; Santos e Wanderley²4; Brasil8; Madeiro25; Rodrigues ${ }^{\mathbf{2 6}}$. 
Sob essa perspectiva, ainda que sejam diversas as causas que levam à ocorrência de incidentes envolvendo barragens, sua dimensão organizacional revela que algumas dessas decisões são claramente erros de projetos; outras são julgamentos que podem ter sido considerados aceitáveis em determinado momento, mas que provaram ser catastróficos quando conjugados com outros eventos ou situações ${ }^{13}$. Ademais, isso se combina com a baixa capacidade do Estado brasileiro de regular e fiscalizar os sistemas sociotécnicos complexos que requerem conhecimento, recurso e autonomia de atuação. Assim, esses processos estão relacionados com as concepções do projeto e/ou ausência de atualização; do licenciamento; da fiscalização, monitoramento e gerenciamento dos riscos (técnicos ou organizacionais) que foram tomadas e identificadas ao longo do tempo ${ }^{\mathbf{1 4}}$.

\section{Barragens e saúde pública: riscos potenciais}

A segurança de barragens é um processo que abrange desde a sua concepção até seu descomissionamento e deve ser compor desde o planejamento e o projeto, da construção à operação, considerando, nesse contexto, sua área de impacto direto e indireto, em caso de colapso. As consequências na saúde pública e no bem-estar são diversos, não se limitam apenas ao limite geográfico em que estão situados e apresentam efeitos de longo prazo e de difícil gestão e avaliação.

As ações de preparação para atuação em desastres tomam por base a tipologia de evento com seus potenciais efeitos, inclusive sobre a saúde, envolvendo a produção de orientações técnicas, educação em saúde voltada aos profissionais do Sistema Único de Saúde (SUS) e à população, articulação entre os atores envolvidos no processo de gestão do risco, incluindo a comunidade. No entanto, os desastres possuem características singulares em cada ocorrência, mesmo quando da mesma tipologia, o que leva à necessidade de análise da situação caso a caso para a adoção das medidas necessárias ao evento, tanto aquelas de resposta imediata quanto as que perduram ao longo do tempo, no processo de recuperação ${ }^{15,19}$.

É inegável que a construção de uma barragem pode trazer benefícios socioeconômicos, no entanto, também apresenta riscos significativos e impactos de modo sistêmicos ao meio ao qual se integra. O impacto na saúde humana e sobre os sistemas sociais e ambientais é precisamente o que torna esses empreendimentos deletérios à sociedade. A partir do momento que as barragens falham, apresentam o potencial de causarem tragédias pessoais e sofrimento coletivo, altos níveis de mortalidade e morbidade, impactos diretos e indiretos no progresso político, social e econômico, criando ciclos viciosos de vulnerabilidade; favorecem condições para o surgimento de doenças e agravos em saúde, bem como afetam as condições de vida ${ }^{15}$. No quadro 3, são apresentados os principais impactos das barragens e as principais causas e danos associados ao rompimento. 
Quadro 3. Impactos de implementação de grandes barragens e causas e efeitos comuns de rompimento

\section{Impactos}

- prejudicaram os ambientes naturais;

- inundaram áreas de desova de peixes;

- inibiram a migração sazonal de peixes;

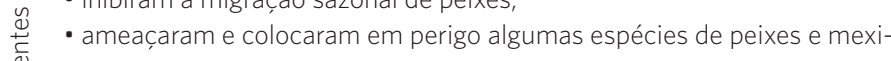
Ihões;

- provocaram alterações profundas na fauna e na flora locais;

- produziram alterações nos processos geomorfológicos em consequência

de mudanças na dinâmica fluvial;

- afetaram os regimes hidrológicos de superfície e subterrâneo;

- contribuíram para o agravamento dos elevados défices sedimentares;

- contribuíram para o desequilíbrio ou desaparecimento de ecossistemas; e,

- podem ter inundado sítios arqueológicos ou cemitérios ancestrais.
Principais causas de colapso-rompimento

- deficiências no processo de construção;

- manutenção inadequada;

- operação ineficiente;

- sabotagem e operações militares;

- exploração inadequada da represa;

- acúmulo de água de maiores proporções ou de importante sequência de cheias;

- ocorrência de um sismo intenso, podendo influenciar a hidrodi-

nâmica;

- escorregamentos de encostas e/ou do talude da represa;

- degradação da estabilidade ou resistência do corpo da barragem, das fundações e/ou das encostas da represa.

- as represas inundam grandes áreas, resultando em perdas de terrenos, culturas e, eventualmente, povoamento;

- podem obrigar o deslocamento de pessoas em busca de novas habitações;

- promovem efeitos erosivo e de instabilidade das encostas marginais da represa por ações do vento, das ondas e das correntes;

- produzem gases de efeito de estufa, como o metano e o dióxido de car-

bono;

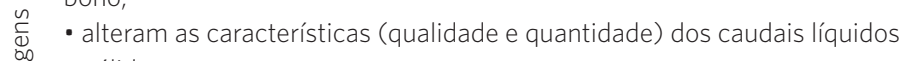

$\underset{0}{\infty}$ e sólidos;

•

ก

O - retêm elevadas quantidades de sedimentos e nutrientes;

- podem promover doenças se não forem geridas adequadamente;

- reduzem a capacidade de transporte do escoamento;

- alteram a morfologia dos leitos aluvionares;

- contribuem para o aparecimento de nevoeiros e neblinas, bem como para

alterações locais do regime de precipitação;

- contribuem para a redução das amplitudes térmicas diárias e anuais;

- contribuem para a redução da frequência de geadas; e,

- as represas podem induzir sismos.

\section{Principais efeitos negativos à saúde, meio ambiente e condição socioeconômica, em caso de incidente}

\section{Curto prazo (dias, semanas e meses)}

- óbitos diretamente associados;

- afogamentos e sufocamento;

- impacto social (desabrigados, deslocados, desalojados);

- comprometimento dos serviços de provisão de alimentos e água potável;

- doenças transmitidas pelo uso de água não adequada para o consumo humano;

- doenças respiratórias e contaminação com a transformação da lama em fonte de poeiras e material particulado;

- dermatites;

- impactos psicossociais e na saúde mental (ansiedade, transtornos mentais, depressão);

- parasitoses, mordeduras e picadas;

- arboviroses (dengue, zika, chikungunya, febre amarela, malária);

- hipertensão arterial sistêmica.

\section{Médio e longo prazo (meses e anos)}

- intoxicação e danos à saúde resultado da toxicidade;

- doenças respiratórias e contaminação com a transformação

da lama em fonte de poeiras e material particulado;

- impactos psicossociais e na saúde mental;

- doenças transmitidas pelo uso de água não adequada para o consumo humano;

- doenças respiratórias e contaminação com a transformação da lama em fonte de poeiras e material particulado;

- Dermatites;

- arboviroses (dengue, zika, chikungunya, febre amarela, malária);

- desfechos negativos à saúde derivado das águas, solo e cadeia alimentar contaminados. 


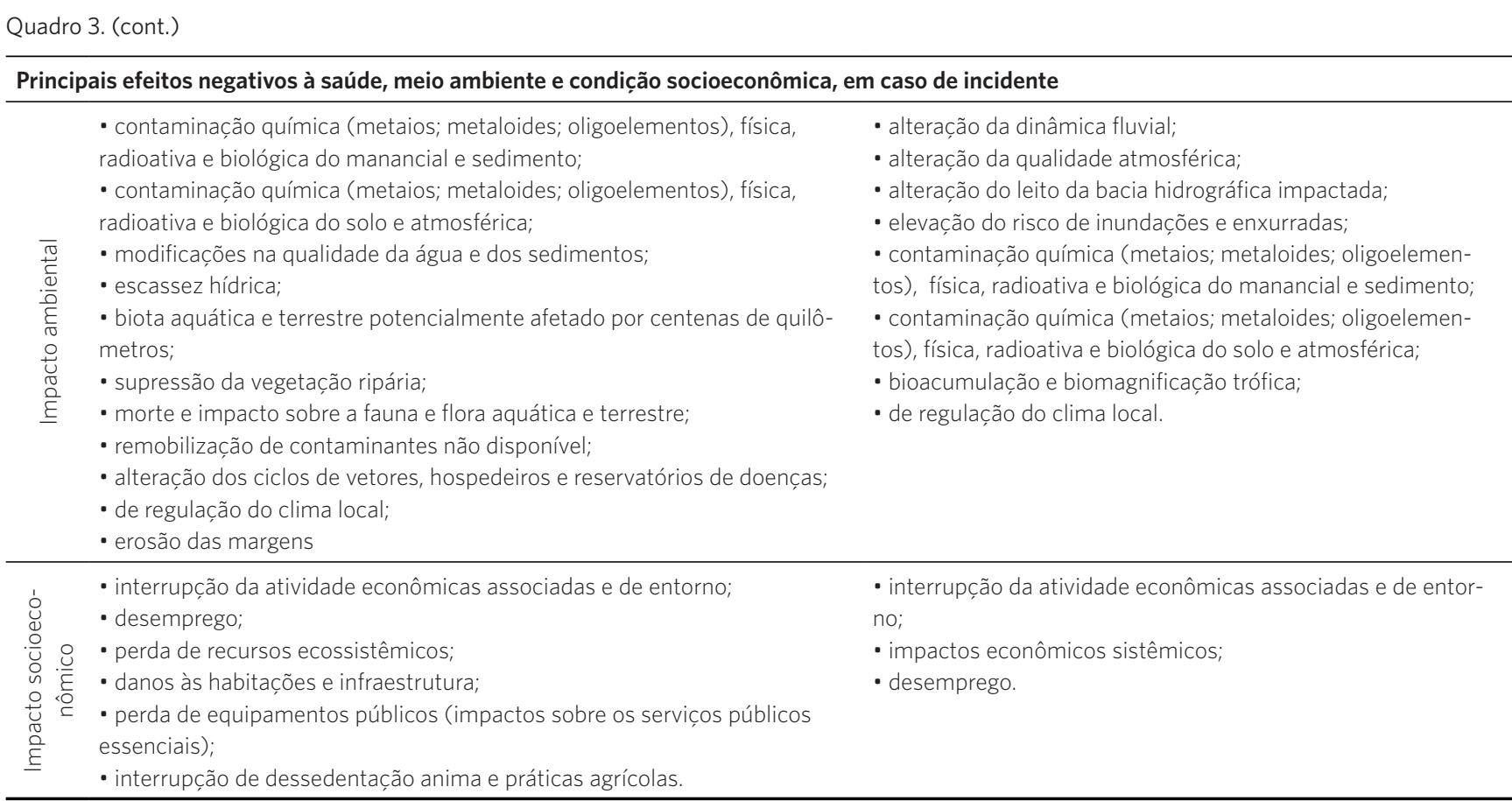

Fonte: Adaptado de Do Carmo 16; Kossoff27; Freitas, Barcellos, et al.2.

Do Carmo ${ }^{16}$ ainda reforça algumas questões-chave relacionadas com a gestão de riscos de desastres, incluindo, entre elas, as estratégias de adaptação, em que aponta quatro como as principais: a) a avaliação de risco deve ser utilizada como uma ferramenta para a tomada de decisão; b) os fatores ambientais, sociais e econômicos devem incorporar o desenvolvimento de estratégias de gestão do risco; c) a compreensão do risco de eventos extremos é uma questão transversal e multidisciplinar; e d) as lições aprendidas, a partir da ocorrência de desastres anteriores, têm mostrado que a inclusão da gestão do risco em todas as etapas do processo que envolvem barragens (gestão end-to-end) é questão indispensável para eficiência.

Uma compreensão sistêmica da concepção ao descomissionamento até a estratégias de enfrentamento de situações de emergências relacionadas com este tipo de empreendimento deve ser o foco de atuação das ações de RRD. Bonatti e Carmo ${ }^{\mathbf{1 7}}$ (apud Hogan) chamam atenção para eventos que influenciaram uma nova percepção sobre a degradação ambiental no mundo, como a contaminação química por mercúrio em Minamata (Japão) em 1956 e do Love Canal em Nova Iorque na década de 1980, a nuvem tóxica em Seveso na Itália em 1976, o vazamento de gás em Bhopal na Índia em 1984, o desastre nuclear de Chernobyl em 1986. Os autores apontam que além de impactos diretos, por meio de danos físicos, econômicos e sociais, os danos em longo prazo são dificilmente mensuráveis, dado que os riscos e danos de eventos continuam a se ampliar no tempo e no espaço.

Segundo Freitas ${ }^{\mathbf{1 8}}$, esses desastres tecnológicos revelam algumas características que devem ser destacadas. A primeira é pela razão de envolverem instalações fixas, que processavam materiais perigosos, localizadas muito próximas de áreas densamente povoadas. A segunda é que, para além do ponto de origem do desastre, os danos e degradação ambiental, bem como agravos à saúde, ampliam-se no espaço e tempo. A terceira se refere ao fato que, mesmo com 
tecnologias de segurança disponíveis, o desenvolvimento de sequência de eventos incontroláveis no processo de produção acaba por resultar em desastres, apresentando liberação de produtos perigosos para a saúde e o meio ambiente para além dos muros dessas instalações. Como observa o autor, incidentes e acidentes nesses tipos de instalações são mais frequentes do que a imagem pública que as empresas envolvidas procuram divulgar, de modo que, em todos esses desastres, investigações posteriores demonstraram falhas e até mesmo degradação nos sistemas de segurança e prevenção de acidentes. Por fim, em alguns casos, como em Seveso e Bophal, Three Mile Island e Chernobyl, houve demora na obtenção de informações precisas sobre os produtos envolvidos e seus riscos e efeitos para a saúde pública, resultando na demora em ações de emergência para redução da exposição e riscos das populações atingidas, bem como comprometimento do tratamento inicial dos diretamente expostos aos produtos perigosos.

Do ponto de vista da saúde pública, para além dos óbitos imediatos que muitos desses desastres podem provocar, representam mudança dos cenários de riscos passados. Isso inclui tanto os relacionados com os sistemas de risco intensivos, ligados ao projeto e operações das atividades tecnológicas de mineração e suas barragens, assim, como todos os procedimentos de controle e gestão de risco, mas também à situação de saúde; modificação das condições de vida; riscos ambientais. Por outro lado, a partir do momento em que ocorrem, produzem novos cenários de riscos ${ }^{19}$ e problemas extensivos ${ }^{\mathbf{2 0}}$, como os problemas ambientais e à saúde, que se estendem no espaço, atuam em unidades interescalares - indo do local ao regional-, assim como no tempo, produzindo uma multiplicidade e sobreposição de situações de exposições, riscos e efeitos sobre a saúde, que vão dos imediatos aos de longo prazo, e a depender dos produtos envolvidos, apresentando potencial de causar efeitos carcinogênico, mutagênico e teratogênico.

Essas características estiveram presentes em dois dos mais graves desastres tecnológicos em barragens de mineração no mundo, ambos ocorridos no Brasil. O primeiro envolvendo a empresa Samarco em 2015, com 19 óbitos, sendo que, desses, 14 (74\%) de trabalhadores, envolvendo 50 milhões de metros cúbicos de lama de rejeitos, atingindo 36 municípios em uma extensão de $650 \mathrm{~km}$ ao longo do rio Doce. O segundo envolvendo a Vale S.A., em 2019, entre óbitos e desaparecidos, totaliza cerca de 270 vítimas, constituindo o maior acidente de trabalho já ocorrido no Brasil. Além de vítimas fatais e atingidos, esse desastre envolveu 13 milhões de metros cúbicos de lama de rejeitos, atingindo, ao menos, 18 municípios em uma extensão centenas de quilômetros ${ }^{6}$. No quadro 3 , apresentamos os possíveis cenários de risco para além dos dados gerados no curto prazo, incluindo contaminação e alterações ambientais (impactos sobre a biodiversidade e alterações dos ciclos de vetores, hospedeiros e reservatórios de doenças), como também a alteração abrupta da organização social e dos modos de viver e trabalhar historicamente constituídos nos territórios, com efeitos sobre a saúde².

Quando considerado o caso da Vale S.A. em Brumadinho (MG), a magnitude com que o evento se deflagrou resultou em danos à saúde e aumento de psicopatologias. O setor saúde municipal necessitou reorganizar seu fluxo assistencial e adaptar-se às novas necessidades de saúde pós-desastre. Embora o total de feridos e lesionados registrados tenha sido praticamente nulo, após as primeiras 72 horas até o fim do primeiro mês, registraram-se diversas reações psicológicas na população, sendo as mais frequentes de caráter agudo, além do aumento do consumo de álcool, conflitos interpessoais e situações de violência ${ }^{\mathbf{2 1}}$.

Foram contratados mais de 80 profissionais para dar conta dos atendimentos, houve ampliações de ações no âmbito de atenção em saúde, atenção psicossocial e ações de urgência e emergência para resgate e tratamento que envolvia não só populações afetadas diretamente, mas também fortalecimento de ações de vigilância em saúde de longo prazo. O uso de ansiolítico aumentou em $80 \%$; de antidepressivos, 
em 60\%; e o aumento dos gastos em saúde representou acréscimo de 1,5 milhão por mês. A implementação de um conjunto integrado de ações de prevenção, imunização, vigilância em saúde (epidemiológica e sanitária) e atenção em saúde permitiu ofertar à população serviços estratégicos para reduzir os danos do evento. Essa nova organização do setor saúde contou com o apoio dos três níveis da federação em ações compartilhadas, inclusive com outros atores externos ao SUS, permitindo assegurar a RRD não só ao município de brumadinho, mas também aos demais município afetados indiretamente pelo desastre ${ }^{2}$.

Mesmo o rompimento de barragens que não registraram óbitos associados apresentou o potencial de causar significativos impactos sobre o processo de saúde e doença das populações afetadas. $\mathrm{O}$ recente transbordamento da Barragem do Quati, em 2019, localizada no município de Pedro Alexandre (BA), causou elevação dos níveis do Rio do Peixe e desencadeou uma onda de inundação/enxurrada atingindo a cidade à jusante de Coronel João Sá. Registraram-se diversos danos materiais, modificação ambiental e social, com prejuízos de equipamentos públicos e 430 famílias (1.720 pessoas) desalojadas/desabrigadas.

A partir da organização do Comitê Operativo de Emergência do setor saúde, foram disparadas ações de identificação e cadastros dos afetados; acolhimento e organização dos abrigos; triagem e inventário de doações; comunicação de risco; e imunização em larga escala. As ações foram integradas a partir das equipes municipais e estaduais de vigilância em saúde ambiental (vigilância epidemiológica e sanitária); atenção básica; capacitação dos agentes comunitários de saúde para desempenho de ações de promoção à saúde de longo prazo. Disponibilizaram-se leitos nos centros ambulatoriais de referência (Hospital Municipal de Antas e Hospital Santa Tereza) para ao atendimento de urgência e emergência; ampliou-se o efetivo das Equipes de Saúde da Família (ESF) em consonância com elevação da demanda de atendimentos.
Para a saúde pública, os desafios também não são pequenos, pois, ainda que barragens apresentem o potencial de causar impactos associados durante todo seu ciclo de vida, incidentes abrangendo esse tipo de estrutura envolvem óbitos imediatos e danos à saúde no médio longo prazo. Mesmo diante desse fato, os dados sobre acidentes com barragens estão dispersos em diversas bases, com informações incompletas e com baixa transparência sobre os motivos das ocorrências e impactos associados, o que resulta na sensação de que não se evolui com as lições aprendidas com desastres do passado para evitar desastres futuros.

\section{Fiscalização como requisito básico para o planejamento de ações: nós críticos}

Tendo como referência o Marco de Sendai, muitas vezes não é o fenômeno natural ou o desenvolvimento tecnológico que determinam a ocorrência de desastre, mas sua combinação com as condições de vulnerabilidade, as diferentes formas e vias de exposição, bem como as capacidades institucionais de prevenção e respostas das sociedades, envolvendo da antecipação e alerta aos processos de recuperação e reconstrução como fatores determinantes para a sua ocorrência ${ }^{22}$. Se, no passado, as instituições visavam apenas minimizar as consequências dos desastres, atualmente, suas atividades também incluem, por meio de trabalhos preventivos, a RRD.

Se, por um lado, temos assistido experiências exitosas de integração da gestão de riscos de emergências e desastres aos cuidados primários, secundários e terciários (como vimos nos tópicos anteriores), por outro, ainda há necessidade explícita de entender o risco desse tipo de desastres envolvendo barragens por meio da ótica de RRD. Na tabela 1, demonstramos que das 24.092 barragens que estão registradas no Snisb, apenas $58 \%$ dessas barragens estão regularizadas. Até o momento, $85 \%$ das barragens não foram avaliadas quanto à categoria de risco e 77,3\% ainda não foram 
classificadas quanto ao DPA. São 18.446 (76\%) e $9.584(39,7 \%)$ barragens que não possuem informação de altura e capacidade armazenada respectivamente.

A ausência de informações suficientes para a tomada de decisão é um fato preocupante no cenário de risco nacional, pois o conhecimento se a barragem se submete ou não à PNSB é o trabalho básico de todas as entidades fiscalizadoras. Passados nove anos após a implementação da PNSB, essa obrigação deveria estar praticamente finalizada. Cabe frisar que a legislação vigente prevê, mediante o Plano de Segurança de Barragem, inspeções regulares ou especiais (órgãos fiscalizadores), Revisões Periódicas de Segurança de Barragem (RPSB) (art. $9^{\circ}$ ) (por parte do empreendedor) e a elaboração de Planos de Ação de Emergência (PAE), sempre que a barragem for classificada como de DPA alto (art. 11). Segundo ANA, atualmente $27 \%$ das barragens são submetidas à PNSB, desse total, 22,4\% foram inspecionadas no ano de 2017 e 17\% definiram rotina de RPSB. Em relação ao PAE, apenas $25 \%$ das barragens com DPA alto apresentaram à sociedade planos de emergência ${ }^{8}$.

Em relação aos órgãos fiscalizadores, no cenário nacional, cerca de $55 \%$ desses órgãos não possuem uma equipe com atribuição formal e exclusiva para atuar em segurança de barragens. Quando considerado o quantitativo de profissionais integrando as equipes de fiscalização, verifica-se que $61 \%$ delas (19 fiscalizadores) possuem teoricamente quantitativo adequado para desempenho das atividades, e 39\% (12 fiscalizadores) não. Do total do orçamento empenhado para gestão de segurança de barragem, foi aplicado $\mathrm{R} \$$ 34 milhões em operações de manutenção e recuperação de barragens no ano de 2017, valor correspondente a $23 \%$ do previsto pelo governo federal e $73 \%$ do previsto dos governos estaduais. Chama atenção que 45 barragens, em 2017, foram consideradas em risco, em sua maioria, devido a problemas de conservação. O número pode ser ainda maior: considerando o total de barragens submetidas à PNSB, as consideradas de maior dano associada em caso de incidentes, apenas 17\% são, em média, vistoriadas anualmente'.

As tragédias mineiras expõem uma dificuldade inerente aos municípios mineradores. No Brasil, as duas barragens da Vale que se romperam possuíam planos de emergências que existiam só no papel, e ficou comprovado que os sistemas de alerta e alarme eram inexistentes (em Mariana) e inefetivos (Brumadinho)6. Para além dos setores envolvidos no licenciamento e fiscalização, a participação proativa dos setores de saúde, trabalho, proteção e defesa civil, assim como dos trabalhadores, comunidades e organizações da sociedade civil, certamente impediria alguns dos erros cometidos. Além disso, é necessário que planos, alertas e alarmes não sejam tratados somente como obrigações legais, mas que sejam fruto de um efetivo e transparente processo de planejamento, com a participação da sociedade, fortalecendo as capacidades de preparação e respostas dos municípios por intermédio da realização de exercícios regulares. Essa mudança evitaria as situações recentes; as vivenciadas nos municípios mineiros de Ouro Preto, Nova Lima e Barão de Cocais, entre outros, que de uma hora para outra passaram a não ser mais considerados seguros.

Por fim, se até aqui privilegiamos a análise tomando como referência as ameaças em nosso território, devemos considerar também as condições de vulnerabilidade que estão na raiz do próprio conceito de desastres. De Marchi e Ravetz ${ }^{\mathbf{2 3}}$ apontam aspectos importantes para combinar a RRD com a governança. Nessa perspectiva, é necessário não só estruturar os órgãos fiscalizadores para desempenho de sua função, mas também combiná-los com participação da sociedade e transparência pública como aspectos básicos. Destacamos três princípios apontados pelos autores: 1 ) o conhecimento partilhado entre as instituições nos processos de gestão - reconhecer a necessidade de incorporação de diferentes tipos de conhecimento, a diversidade de atores e experiências na definição do risco significa a efetiva participação de trabalhadores e comunidades 
nos processos de gestão dos riscos de desastre, além de representações da sociedade civil organizada; 2) a congruência dos projetos, planos e ações de prevenção e mitigação de riscos, bem como de resposta e recuperação em desastres, considerando um conjunto de diferentes atores e escalas espaciais e temporais de atuação; 3) a confiança, como condição fundamental, o que envolve não só uma ação integrada dos diferentes órgãos de governo, mas também a transparência das informações e decisões associada à uma participação efetiva dos diferentes atores, sejam institucionais ou leigos.

Não é só a inexistência de regulação para os direitos dos atingidos, mas a forte regulação dos direitos das empresas que vem gerando assimetria entre as partes. Atualmente, existe um enorme esforço social para assegurar e estabelecer os direitos empresariais, em detrimento da garantia dos direitos das populações atingidas. Sem que ocorra transparência e efetiva participação da sociedade nos arranjos institucionais, pouco avançaremos na RRD no País. Dessa forma, possivelmente experimentaremos novos desastre no setor da mineração, ou mesmo, agravamentos dos desastres já em curso.

\section{Considerações finais}

O presente trabalho procurou demonstrar que a implementação da PNSB se constitui um dispositivo com potencialidade de instituir mudanças no cenário nacional de gestão de risco de desastre. O Snisb e o arranjo institucional implementado a partir desse dispositivo são capazes de cumprir seu papel, por integrar e unir diversos dispositivos institucionais na fiscalização, monitoramento e intervenção, e por trazer a discussão ao cenário nacional de gestão de barragens.

Constituiu-se um esforço de sistematização e discussão dos temas relacionados com a gestão de risco de barragens no cenário nacional, bem como das políticas adotadas para o seu enfrentamento. Embora cada um desses temas não possa ser dissociado dos processos de desenvolvimento econômico e social, e das estruturas políticas existentes, somente na última década tornaram-se foco na agenda nacional com vistas aos desafios de constituir políticas públicas para a prevenção e gestão dessas ocorrências. Nesse sentido, tentamos reconstituir o cenário de risco, procuramos registrar a importância da efetivação da PNSB e as diferentes visões que devem compor as discussões no estabelecimento de medidas gerenciamento desses eventos e sua importância para a tomada de decisão

Nesse ponto, o Brasil não só avançou desenvolvendo políticas específicas ao tema, mas também desenvolveu aparatos institucionais e avanços em relação às necessidades identificadas. Por ser tratar de um arcabouço recente e da necessidade de envolvimento de instituições em diferentes níveis federativos, os resultados assegurados até o momento ainda se demonstram insuficientes, no entanto, possibilitam vislumbrar um horizonte de trabalho futuro ainda a ser concluído.

Ao setor saúde, ainda resta a efetiva implementação dos Princípios de Sendai em sua atuação e nas políticas e ações de redução de risco de desastre em barragens, concernindo ao setor: 1) que as políticas de saúde sejam aplicadas de forma articulada e intersetorialmente com os diversos órgãos de licenciamento, fiscalização e controle prospectivos e preventivos de redução dos riscos de desastres, sendo imprescindível a participação da saúde do trabalhador e a saúde de modo geral, pois, certamente, esses atores terão muito a contribuir para a gestão de risco e para a PNSB; 2) é necessário que o setor saúde melhore as ações de preparação e resposta, ampliando sua capacidade de atuação institucional, não só no curto prazo (dias, semanas e meses), no médio prazo, mas também no longo prazo (anos e décadas), ofertando serviços estratégicos para atendimento das necessidades de saúde das populações atingidas; 3 ) ainda nos resta que a própria constituição das políticas do setor saúde mude de perspectiva. Temos diversas unidades de saúde em áreas de risco 
e, provavelmente, na rota das barragens; vulnerabilidade que fragiliza a capacidade de resposta do setor saúde, inclusive a integridade física e psicológica dos profissionais de saúde que nelas atuam.

Estudos como este são relevantes na medida em que permite repensar a gestão de risco de desastre no cenário nacional, sem, contudo, deixar de evidenciar as múltiplas vulnerabilidades do sistema nacional de gerenciamento de risco. Carecemos ainda, no âmbito nacional, da combinação de atividades de preparação e investimento em formação de
RRD, sem, contudo, negligenciar a atuação das instituições sobre os fatores subjacentes relacionados, direta e indiretamente, com as emergências, sendo necessário avançar em diversos pontos para efetiva gestão de risco, como demonstrado.

\section{Colaboradores}

Silva EL (0000-0003-4608-5946)* e Silva MA (0000-0002-6021-4794) contribuíram igualmente para a elaboração do manuscrito.

\section{Referências}

1. Agência Nacional das Águas; Ministério do Meio Ambiente. Classificação de Barragens: Melhores Práticas Nacionais e Internacionais [Documento Técnico]. Brasília, DF: ANA; 2013.

2. Freitas C, Barcellos C, Asmus C, et al. Da Samarco em Mariana à Vale em Brumadinho: desastres em barragens de mineração e Saúde Coletiva. Cad. Saúde Pública. 2019; 35(5):e00052519.

3. Brasil. Lei ${ }^{\circ} 12.234$, de 20 de setembro de 2010. Estabelece a Política Nacional de Segurança de Barragens destinadas à acumulação de água para quaisquer usos, à disposição final ou temporária de rejeitos e à acumulação de resíduos industriais, cria o Sistema Nacional de Informações sobre Segurança de Barragens e altera a redação do art. 35 da Lei $n^{\circ}$ 9.433, de 8 de janeiro de 1997, e do art. $4^{\circ}$ da Lei no 9.984 , de
17 de julho de 2000. Diário Oficial da União. 21 Set 2010.

4. Comitê Brasileiro de Barragens. A história das barragens no Brasil, Séculos XIX, XX e XXI: cinquenta anos do Comitê Brasileiro de Barragens. Rio de Janeiro: CBDB; 2011.

5. Wanderley L, Mansur M, Milanez B, et al. Desastre da Samarco/Vale/BHP no Vale do Rio Doce: aspectos econômicos, políticos e socioambientais. Ciênc. Cultura. 2016; 68(3):30-35.

6. Freitas C, Silva M. Acidentes de trabalho que se tornam desastres: os casos dos rompimentos em barragens de mineração no Brasil. Rev. bras. med. trab. 2019; 17(1):21-29.
${ }^{*}$ Orcid (Open Researcher and Contributor ID). 
7. Azam S, Li Q. Tailings dam failures: a review of the last one hundred years. Geotech. News. 2010; 28(4):50-54.

8. Agência Nacional das Águas. Relatório de segurança de barragens 2017. Brasília, DF: ANA; 2018.

9. Davies M, Martin T. Mining market cycles and tailings dam incidents. In:Proceedings of the 13th International Conference on Tailings and Mine Waste, Banff, Alberta 2009. Jan, Fort Collins. Colorado: US. Balkema Publishers, 2009. p. 3-15.

10. Agência Nacional das Águas. Relatório de Segurança de Barragens 2017: Cadastro de Barragens (planilha Excel) [internet]. Brasília, DF: Agência Nacional das Águas; 2017 [acesso em 2019 jun 6]. Disponível em: http://www.snisb.gov.br/portal/snisb/relatorio-anual-de-seguranca-de-barragem/2017.

11. Bowker LN, Chambers DM. The risk, public liability, \& economics of tailings storage facility failures. Earthwork Act. 2015; (24):1-56.

12. Chambers DM, Higman B. Long term risks of tailings dam failure [internet]. Montana: Center for Science in Public Participation, Bozeman; 2011. [acesso em 2019 jul 10]. Disponível em: http://www.csp2.org/files/reports/Long\%20Term\%20Risks\%20of\%20Tailings\%20Dam\%20Failure\%20-\%20Chambers\%20 \%26\%20Higman\%20Oct11-2.pdf.

13. Pate-Cornell M. Learning from the Piper Alpha Accident: A Postmortem Analysis of Technical and Organizational Factors. Risk Analysis. 1993; 13(2):215-232.

14. Llory M, Montmayeut R. O acidente e a organização. Belo Horizonte: Fabrefactum; 2014.

15. Organização Pan-Americana da Saúde. Desastres Naturais e Saúde no Brasil. Brasília, DF: Ministério da Saúde; 2014. [Série Desenvolvimento Sustentável e Saúde 2].

16. Carmo JSA. Grandes barragens: vulnerabilidades e riscos. Coimbra: Universidade de Coimbra; 2013.

17. Bonatti TF, Carmo RL. Desastres tecnológicos: revisitando a discussão sobre a questão dos eventos de contaminação a partir da relação entre população, espaço e ambiente [internet]. In: Anais do $20^{\circ}$ Encontro Nacional de Estudos Populacionais, 2016. 2017. p. 1-21. [acesso em 2019 jul 10]. Disponível em: www.abep.org.br/publicacoes/index.php/ anais/index.

18. Freitas C, Gomez C. Análise de riscos tecnológicos na perspectiva das ciências sociais. Hist. cienc. saúde-Manguinhos. 1996; 3(3):485-504.

19. Narváez L, Lavell A, Pérez Ortega G. La Gestión del Riesgo de Desastres. La Paz: Cebem; 2009.

20. Wynne B. Unruly Technology: Practical Rules, Impractical Discourses and Public Understanding. Social Stud. Scien. 1988; 18(1):147-167.

21. Noal D, Rabelo I, Chachamovich E. O impacto na saúde mental dos afetados após o rompimento da barragem da Vale. Cad. Saúde Pública. 2019; 35(5):e00048419.

22. United Nations Office for Disaster Risk Reduction. Sendai framework for disaster risk reduction 20152030. 3. ed. United Nations World Conference on DRR. Sendai: United Nations; 2015.

23. Marchi B, Ravetz J. Risk management and governance. Futures. 1999; 31(7):743-757.

24. Santos RS, Wanderley LJ. Dependência de barragem, alternativas tecnológicas e a inação do estado: repercussões sobre o monitoramento de barragens e o licenciamento do Fundão. In: Zonta M, Trocate C, organizadores. Antes Fosse Mais Leve a Carga: Reflexões sobre o desastre da Samarco/Vale/BHP Billiton. Marabá: Iguana Editorial. 2016. [Col. A Questão Mineral no Brasil. v. 2]. p. 87-137.

25. Madeiro C. Brasil registrou 65 mortes em 9 incidentes com barragens entre 2001 e 2018 [internet]. Uol. 
2019 fev 2. [acesso em 2019 jul 24]. Disponível em: https://noticias.uol.com.br/cotidiano/ultimas-noticias/2019/02/03/brasil-registrou-65-mortes-em-9-incidentes-com-barragens-entre-2001-e-2018.htm.

26. Rodrigues L. Desde 2000, o Brasil tem um rompimento de barragem a cada dois anos [internet]. Jornal O Sul. 2019 jan. 29. [acesso em 2019 ago 26]. Disponível em: https://www.osul.com.br/desde-2000-o-brasil-tem-um-rompimento-de-barragem-a-cada-dois-anos-veja-lista/.

27. Kossoff D, Dubbin W, Alfredsson M, et al. Mine tailings dams: Characteristics, failure, environmental impacts, and remediation. Applied Geochemistry.

2014; (51):229-245.

28. Bahia. Secretaria de Vigilância em Saúde. Relatório do rompimento de barragem de Quati - município Coronel João Sá, 2019. Salvador: Governo Estadual da Bahia; 2019.

Recebido em 29/09/2019

Aprovado em 10/03/2020

Conflito de interesses: inexistente

Suporte financeiro: não houve 